\title{
HACIA UN MODELO DE GESTIÓN DE LOS HUERTOS URBANOS MUNICIPALES: EL CASO DEL PROYECTO RU:RBAN EN LA CIUDAD DE A CORUÑA (PÓSTER)
}

\author{
Matías Pino ${ }^{a}$ \\ ${ }^{a}$ Universidad de Santiago de Compostela.E-mail: matias.pino@ rai.usc.es
}

\begin{abstract}
Resumen
Diversos estudios resaltan que una buena gobernanza de las experiencias de agricultura urbana resulta clave para garantizar su eficacia en el logro de objetivos de desarrollo urbano sostenible. Los huertos urbanos cuentan con un gran potencial para aumentar la resiliencia de las ciudades hacia la sostenibilidad por su multifuncionalidad y por la producción y uso de conocimiento a nivel local; y los gobiernos locales intentan capitalizarlo a partir de políticas de fomento y herramientas concretas para su regulación e implementación. En este sentido, a pesar de que se han realizado notables avances en la investigación de políticas a desarrollar para la implementación de huertos urbanos, aún quedan cuestiones que continúan sin aclararse y requieren mayores investigaciones.

En este trabajo se presenta la experiencia de la ciudad de A Coruña en el proyecto Ru:rban del programa de cooperación territorial europeo URBACT. El proyecto busca transferir las buenas prácticas del modelo de gestión de huertos urbanos de la ciudad de Roma a partir de tres componentes: 1) Desarrollo de capacidades en la gestión de huertos urbanos. 2) Formación profesional de monitores de huertos urbanos. 3) Gobernanza y regulación de huertos urbanos.
\end{abstract}

Palabras clave: gobernanza, gestión, huertos urbanos, sostenibilidad.

\section{Introducción y objetivos}

Los huertos urbanos presentan numerosos beneficios para impulsar el desarrollo urbano sostenible, como la mejora de la biodiversidad, aportes sobre la salud física y mental de las personas y menor energía requerida para conservar los alimentos. Dichos beneficios justifican que cada vez más ayuntamientos se dediquen a promover la agricultura urbana a través de políticas de fomento y herramientas concretas para su regulación e implementación. En este contexto, el desarrollo de un buen modelo de gestión promovido desde los gobiernos locales resulta de gran utilidad para potenciar su impacto y asegurarse de que los objetivos sean coherentes con la estrategia de desarrollo sostenible de la ciudad.

En esta investigación se estudiarán los aspectos más relevantes a tener en cuenta para la gestión de los huertos urbanos municipales, a partir de los resultados de la participación de la ciudad de A Coruña en el proyecto Ru:rban del programa de cooperación territorial europeo URBACT. El programa URBACT funciona como una red de transferencia de buenas prácticas entre las ciudades europeas, con el objetivo de fomentar el desarrollo urbano sostenible. En este caso, el proyecto Ru:rban busca transferir las buenas prácticas de la ciudad de Roma en la gestión de huertos urbanos a otras seis ciudades: A Coruña (España), Caen (Francia), Loures (Portugal), Crakovia (Polonia), Tesalónica (Grecia) y Vilna (Lituania).

El primer objetivo de esta investigación será realizar un análisis con voluntad descriptiva de la experiencia de la ciudad de A Coruña en el proyecto Ru:rban, a partir de los tres elementos trabajados conjuntamente entre las ciudades participantes: 1) Desarrollo de capacidades en la gestión de huertos urbanos. 2) Formación profesional de monitores de huertos urbanos. 3) Gobernanza y regulación de huertos urbanos. A partir de este primer análisis, el segundo objetivo de esta investigación será realizar un análisis con voluntad prescriptiva que recoja los mecanismos municipales a priorizar para la construcción de un modelo de gestión de los huertos urbanos municipales.

\section{Metodología}

La metodología empleada para la investigación incluirá los siguientes aspectos:

- Rastreo de noticias publicadas en medios digitales y webs institucionales del Ayuntamiento de A Coruña.

- Revisión de la ordenanza municipal del Ayuntamiento de A Coruña con la que se regula la puesta en funcionamiento de los huertos urbanos.

- Revisión de los artículos publicados en la web del proyecto Ru:rban.

- Revisión de las comunicaciones realizadas tras las reuniones transnacionales previstas en el proyecto Ru:rban.

\section{Resultados}

Con la intención de modernizar la gestión de sus huertos urbanos, el Ayuntamiento de A Coruña se presentó al proyecto Ru:rban con la creación de tres zonas destinadas para huertos urbanos ecológicos ("ecohuertos") que albergan un total de 218 parcelas en los barrios de Agra do Orzán (112 parcelas), Eirís (77 parcelas) y 
Novo Mesoiro (29 parcelas). Para lograr capitalizar los conocimientos adquiridos en el proyecto, se creó un grupo de trabajo local integrando diversos actores, como las comunidades de usuarios constituidas en cada zona de ecohuertos, ONGs y centros de educación infantil y primaria. El grupo de trabajo local actúa como el motor del proyecto, puesto que en él se debaten diferentes iniciativas para los ecohuertos, y se transfieren los conocimientos adquiridos en las diferentes reuniones transnacionales celebradas en el marco del proyecto Ru:rban (Ayuntamiento de A Coruña, 2021).

A partir de las reuniones del grupo de trabajo, una de las primeras conclusiones que se alcanzaron fue en el elemento central del proyecto sobre desarrollo de capacidades de gestión y organización de los huertos. Sobre este aspecto se entendió necesaria la consolidación de los mecanismos de participación ciudadana y un enfoque de implementación de abajo hacia arriba ("bottom-up"), donde las asociaciones de usuarios jugaran un rol relevante en el diseño y las características de los huertos (García Gomez \& Lema Blanco, 2018). Los procesos de participación ciudadana que había impulsado previamente el Ayuntamiento tuvieron el objetivo de contar con la implicación activa de los vecinos, identificar entidades potencialmente interesadas y definir aspectos clave como la localización y preparación de los huertos. Adicionalmente, los huertos urbanos ya habían sido apoyados por la ciudadanía en los presupuestos participativos que puso en marcha el Ayuntamiento en los años 2016 y 2017 (Ayuntamiento de A Coruña, 2021).

Una segunda decisión que se tomó a partir de las reuniones del grupo de trabajo local fue el establecimiento de cinco huertos escolares repartidos en las tres zonas de ecohuertos. Esta medida fue acordada con los representantes de cinco centros de educación, y fue acompañada por la creación de una red de ecohuertos para conectar el trabajo de los cinco huertos escolares (Ayuntamiento de A Coruña, 2021). Esta red incluye un punto de información que cuenta con personal calificado para asesorar a los usuarios de los huertos escolares, y constituyó el puntapié inicial para la creación de una red de huertos urbanos municipales que permita conectar todas las iniciativas de la ciudad y mejorar la transferencia de experiencias y conocimientos (Ayuntamiento de A Coruña, 2020).

Una tercera decisión que se tomó en el marco de las actuaciones del grupo de trabajo local fue la puesta en marcha de programas de formación. Para el caso de los huertos escolares, se formaron 15 monitores para desarrollar funciones de diseño y ejecución de los huertos escolares, actividades educativas y dinamización de la comunidad escolar (Ayuntamiento de A Coruña, 2021). Para el resto de los huertos se realizaron cursos para formar la figura conocida como "gardeniser", el cual fue una de las principales lecciones aprendidas de la ciudad de Roma. La figura del "gardeniser" es clave en la coordinación entre los diferentes usuarios, y su trabajo no pasa solo por transmitir técnicas de cultivo, sino que también juega un papel clave para garantizar la cohesión social entre los usuarios y la comunicación con el sector público. Para la formación de los "gardenisers" se impartió un curso de 1.000 horas de duración en el ámbito de la agricultura ecológica y la utilización de los huertos con fines educativos, terapéuticos y de integración social (Ayuntamiento de A Coruña, 2021). Adicionalmente a la formación impartida por el Ayuntamiento, los "gardenisers" recibieron formación en el marco del proyecto Ru:rban, participando en cuatro reuniones transnacionales en el año 2019. La formación recibida siguió una metodología de aprendizaje mediante la práctica ("learning by doing”) sobre gestión, toma de decisiones y liderazgo (Hernandez, 2020).

En el marco del tercer pilar del proyecto sobre gobernanza y regulación de huertos urbanos, también se obtuvieron muchas lecciones. Sobre el modelo de gobernanza, se confirmó que los mejores resultados se obtienen cuando las asociaciones gestionan los huertos, en conjunto con una normativa municipal que incorpore derechos y obligaciones. En adición a lo anterior, se determinó la conveniencia de contar con un grupo de trabajo que participe en el proceso de creación de los huertos desde el principio, y también que la figura del "gardeniser" juegue un rol relevante en la gobernanza como coordinador (Hernandez, 2020).

En cuanto a la regulación, se tomaron en consideración algunos aspectos de la regulación de los huertos de la ciudad de Roma para adaptarlos a la regulación de los huertos de la ciudad de A Coruña. En primer lugar, se entendió beneficioso que la asignación de las parcelas incorpore criterios de inclusión de personas vulnerables. En este sentido, la legislación de A Coruña ya cuenta con criterios de asignación que dan prioridad a determinados colectivos, como jubilados, inmigrantes o víctimas de violencia de género (Ayuntamiento de A Coruña, 2018). En segundo lugar, se sugirió que el tiempo de adjudicación de dos años (con posibilidad de prorrogarlo un año más) de las parcelas de A Coruña era bajo, en comparación con la ciudad de Roma que los adjudica por seis años (Hernandez, 2019). En tercer lugar, se constató que el modelo de reforzar el rol de las asociaciones es clave para el éxito, y debe ser tenido en cuenta en la normativa municipal. Sobre este aspecto, la normativa de A Coruña ya estipula que los usuarios de cada zona de huertos urbanos se organicen bajo una asociación para ejercer la interlocución con el Ayuntamiento (Ayuntamiento de A Coruña, 2018). En cuarto y último lugar, se comprendió que todos los aspectos relacionados con el modelo de gobernanza y la inclusión de derechos y obligaciones debe ser recogida en la regulación de los huertos, así como la figura del "gardeniser" y la inclusión de programas formativos (Hernandez, 2019). 


\section{Conclusiones}

Las conclusiones del proyecto se pueden resumir a partir del vademécum para implementar huertos urbanos que se elaboró a partir de una consulta desde la ciudad de Barranquilla para poner en marcha sus huertos urbanos. Este documento se realizó en colaboración con las ciudades socias del proyecto y reúne los principales aspectos que fueron trabajados, constituyendo un resumen a nivel prescriptivo que contiene los primeros pasos para implementar un programa de huertos urbanos.

Sobre los principales contenidos del documento, se menciona la pertinencia de articular mecanismos de participación ciudadana para que los potenciales usuarios sean parte del proceso de creación desde el principio y decidan la ubicación y las características de los huertos. En cuanto al modelo de gobernanza de los huertos, se especifica que los mejores resultados se obtienen bajo una gobernanza colaborativa entre las asociaciones y el gobierno local, donde las asociaciones se encargan de gestionar y mantener los espacios, y el gobierno local se encarga de acondicionar el terreno y generar una normativa con derechos y obligaciones. Por último, se mencionan los beneficios de contar con una figura como la del "gardeniser", que actúe como líder y dinamizador de la comunidad de usuarios (Hernandez, 2020).

\section{Bibliografía}

Ayuntamiento de A Coruña (2018). "Convocatoria de autorizacións para o uso das hortas urbanas da Agra do Orzán, Novo Mesoiro e Parque de Eirís". Boletín Oficial da Provincia da Coruña.

Ayuntamiento de A Coruña (2020). "O concello impulsará a creación dun punto de información para a Rede de Ecohortos Urbanos". Obtenido de https://www.coruna.gal/emprego/gl/detalle/o-concelloimpulsara-a-creacion-dun-punto-de-informacion-para-a-rede-de-ecohortosurbanos/suceso/1453722175172

Ayuntamiento de A Coruña (2021). "Curso de Formación para o Emprego Monitorado de hortos urbanos". Obtenido de https://www.coruna.gal/emprego/gl/ru-rban/curso-de-formacion-para-o-empregomonitorado-de-hortos-urbanos?argIdioma=gl

Ayuntamiento de A Coruña (2021). "Proyecto europeo Urbact III - Ru:rban: Transferencia de Buenas Prácticas sobre EcoHuertos Urbanos". Obtenido de https://www.coruna.gal/emprego/gl/detalle/proyectoeuropeo-urbact-iii-ru-rban-transferencia-de-buenas-practicas-sobre-ecohuertosurbanos/contenido/1453711489599

García Gómez, M. y Lema Blanco, I. (2019). "Ecohortas urbanas: educando e corresponsabilizando a cidadanía na mellora da biodiversidade urbana e o consumo responsable". Connecting Nature.

Hernandez, P. (2020). "Transfer story - how to implement urban gardens”. URBACT.

Hernandez, P. (2020). “The Gardeniser Toolkit is ready!". URBACT.

Hernandez, P. (2019). "The city of A Coruna hosts its $3^{\text {rd }}$ ULG meeting". URBACT. 